\title{
Design and Modeling of Spoof Surface Plasmon Modes-Based Microwave Slow-Wave Transmission Line
}

\author{
Amin Kianinejad, Student Member, IEEE, Zhi Ning Chen, Fellow, IEEE, and Cheng-Wei Qiu, Member, IEEE
}

\begin{abstract}
This paper presents a theoretical model and validates experimentally the microwave slow-wave transmission line (SW-TL) based on spoof surface plasmon (SSP) modes. Equivalent circuit models are first presented for characterizing the SSP structures and developed to serve as an insightful guideline to design the SW-TL at a given cutoff frequency and Bloch impedance. A mode converter connecting a conventional microstrip transmission line to the SW-TL is necessarily proposed to ensure that the quasi-TEM modes of the microstrip line are gradually transformed to the operating TM modes of the SW-TL. The presented schematic of SW-TL paves a promising avenue for the unprecedented interconnector footprint miniaturization of integrated circuits, and the enhanced electromagnetic compatibility, for example, in multilayered monolithic microwave integrated circuits.
\end{abstract}

Index Terms-Equivalent circuits, impedance matching, mode matching, periodic structures, plasmons, transmission line.

\section{INTRODUCTION}

$\mathbf{S}$ URFACE plasmons (SPs) are intensely localized surface waves at the interface between two materials (e.g., a metal-dielectric interface) of opposite signs in real parts of electric permittivities. These waves propagate along the surface of a conductor and decay exponentially in its transverse direction [1]. By significantly reducing the effective wavelength of surface waves, the SPs present high spatial confinement [2]. The SPs have found potential applications in microscopy, solar cells, optics, magneto-optic data storage, and biological sensors [1].

The SPs originally exist at optical regimes [1]. Nevertheless, Pendry et al. showed that spoof SPs (SSPs) can be

Manuscript received August 25, 2014; revised December 11, 2014 and March 28, 2015; accepted April 01, 2015. Date of publication May 04, 2015; date of current version June 02, 2015. The work of A. Kianinejad was supported by Agency for Science, Technology and Research (ASTAR) through a SINGA scholarship.

A. Kianinejad and Z. N. Chen are with the Department of Electrical and Computer Engineering, National University of Singapore, Singapore 117583, and also with Institute for Infocomm Research, Agency of Science, Technology and Research, Singapore, Singapore 138632 (e-mail: amin@nus.edu.sg; eleczn@nus.edu.sg).

C.-W. Qiu is with the Department of Electrical and Computer Engineering, National University of Singapore, Singapore 117583 (e-mail: chengwei.qiu@nus.edu.sg).

Color versions of one or more of the figures in this paper are available online at http://ieeexplore.ieee.org.

Digital Object Identifier 10.1109/TMTT.2015.2422694 observed at microwave frequencies on perforated conducting surfaces [3]. Various structures that support SSP modes have been reported. Shen et al. introduced a comb-shaped metallic structure supporting the surface plasmon modes propagating on electrically ultrathin and flexible films over a long distance in a wide range from microwave to mid-infrared frequencies. They used nearly zero-thickness and comb-shaped metal strips printed onto flexible dielectric films well supporting the SSP modes [4].

Applications of SSPs as waveguides have been reported. Navarro-Cía et al. proposed the SSP structure based on split ring resonators [5]. Another relevant study employed an array of L-shaped metallic elements that were horizontally attached to a metal surface [6]. Navarro-Cía et al. designed a slow-wave transmission line (TL) and splitter by coaxial spoof plasmons [7]. They used a monopole antenna as a source for their transmission line. Gao et al. proposed a frequency splitter based on their ultrathin dual-band SSP structure [8]. Also, double-grating SSP unit cells have been employed to form an ultra-wideband SSP filter [9]. Liu et al. experimentally verified the propagation of higher order SSP modes in a comb-shape strip, which acts as a multiband TL [10]. Pandey et al. took advantage of three-dimensional (3-D) printing technology to fabricate a terahertz (THz) plasmonic waveguide [11].

The term "single wire" TL was first used by Goubau and composed of a dielectric coated wire connected to two coaxial connectors, forming a groundless TL [12]. In other works, planar transitions were introduced for connecting the groundless Goubau line to coplanar waveguides $(\mathrm{CPWs})$ at $\mathrm{THz}$ bands [13], [14]. The coupling between the Goubau line and resonating structures (ring resonators) has been studied [15]. Akalin and Padila examined the $90^{\circ}$ bending of a Goubau line and designed a filter by adding periodic corrugation to a Goubau line [16]. The feeding transition for Goubau loads and power divider/combiner at millimeter-wave ranges was presented [17]. A coplanar transition to a planar Goubau line at millimeter-wave bands was also proposed [18]. Laurette et al. added corrugations to the Goubau line to form a slow-wave TL (SW-TL) at THz regime [19]. Sánchez-Escuderos added dipole antennas to the Goubau line to form a low-loss leaky wave antenna [20]. Ma et al. exploited SSP modes to construct a TL connected to a CPW line [21]. In another study, the multichannel composite SSPs were excited by CPW [22]. Pan et al. applied electrically resonant metamaterials to control the rejection of an SSP-based TL [23]. Liu et al. designed a 
coupler and frequency splitter by their SSP-based TL [24]. Due to presence of the ground plane on both sides of the CPW, this type of TL has a much wider profile than that of a microstrip $\mathrm{TL}$ with the same impedance. Even for the finite ground CPW (FG-CPW), however, the width of the finite ground plane is one of the limiting factors determining the minimum separation of two TLs [25]. Microstrip TLs, on the other hand, with their narrow profile provide a better choice for compact circuit design; however, their noncoplanarity has restrained their adoption in many applications. Thanks to planar configuration and high field confinement, the SSP-based TLs are able to provide the groundless and compact planar waveguides that could be fabricated very close to each other without significant mutual coupling. To employ this new generation of TL in miniaturized microwave circuits, they should be efficiently connected to conventional TLs such as microstrip TLs. To the best of our knowledge, a broadband and efficient transition from an SSP-based TL to a microstrip TL has not been reported so far.

In this paper, an SW-TL coupled to a microstrip TL is designed and fabricated. In Section II, we first propose an equivalent circuit model for the analysis and design of SSP structures operating at microwave bands. In Section III, a mode converter is proposed to connect the SSP structure to a microstrip TL by an efficient conversion between the TM modes of an SSP structure to the quasi-TEM modes of a conventional microstrip TL, where the converter converts both the momentum and polarization of SSP structures. Moreover, it is shown that impedance matching between two types of structures is achieved through periodic SSP unit cells. In Section IV, the design procedure for the proposed mode converter is studied. We discussed the results in Section V and showed that the SSP structure along with the proposed efficient mode converters forms a groundless and low-loss SW-TL.

\section{Circuit Model for Spoof SP Structures}

Establishing an equivalent circuit model is key to the analysis and thereby the design of the characteristics of a microwave structure. A model is proposed to offer the general understanding of SW-TL and facilitate further advanced engineering designs and optimizations with desired performance. By modeling one unit cell of a periodic structure using a circuit model, the network parameters such as scattering and transmission matrix can be calculated. These parameters, in return, are helpful in calculating the dispersion curve and Bloch impedance of a periodic structure.

Here, the model of a single conductor TL is proposed by using circuit elements. The current in this circuit model is defined as the current on the conductor, similar to the definition of the current in a two-conductor TL. Consequently, the equivalent inductor formulas in two-conductor TLs are applied in this model [26]. The voltage definition for a single-conductor TL is slightly different from conventional models. In two-conductor TLs, the voltage is defined as the voltage difference between the core conductor and the ground plane. In a single-conductor $\mathrm{TL}$, there is no ground plane; however, we still can consider a ground plane very far from the structure and the voltages can be defined as the voltage difference between the TL and infinity.

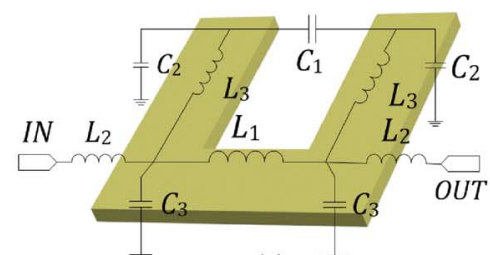

(a)

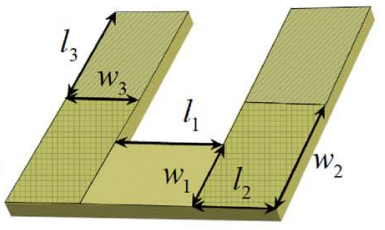

(c)

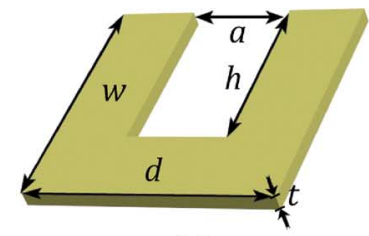

(b)

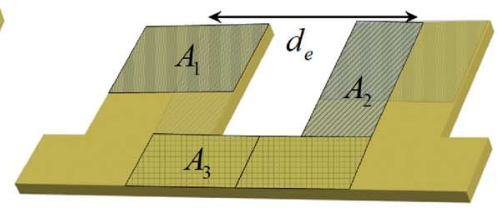

(d)

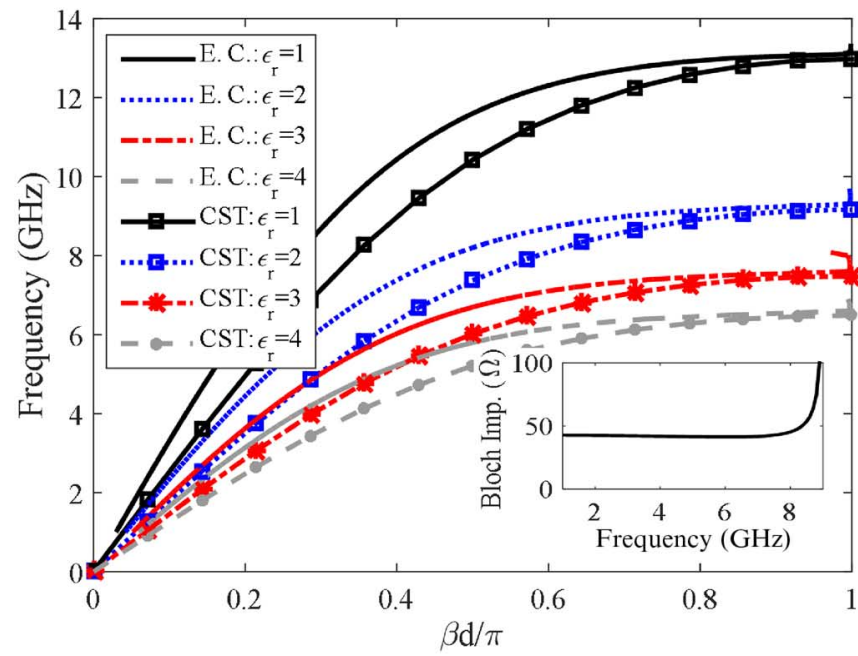

(e)

Fig. 1. (a) Unit cell of the SSP structure along with the equivalent circuit model. (b) Dimensions of the SSP structure. (c) Dimensions of the equivalent inductors (d) Dimensions of the equivalent capacitors. (e) Dispersion curves for the SSP in four background media. Inset: Bloch impedance calculated for the SSP unit cells on Rogers $40031.52 \mathrm{~mm}$.

This definition is applied in calculating $C_{2}$ and $C_{3}$ in Fig. 1(a). These capacitances also model the leaky currents from the TL to the infinite ground, similar to the leaky current in double-conductor TLs.

For periodic structures in general, the following relation exists between input $\left(V_{\mathrm{n}}, I_{\mathrm{n}}\right)$ and output $\left(V_{\mathrm{n}+1}, I_{\mathrm{n}+1}\right)$, i.e., voltages and currents of the $n$th unit cell:

$$
\begin{aligned}
& V_{\mathrm{n}+1}=V_{\mathrm{n}} e^{\gamma p} \\
& I_{\mathrm{n}+1}=I_{\mathrm{n}} e^{\gamma p}
\end{aligned}
$$

where $p$ is the period length. The complex propagation constant is $\gamma=\alpha+i \beta$, where $\alpha$ and $\beta$ are the decay and propagation constants, respectively. The transmission parameters of one unit cell are defined in an ABCD matrix as follows [27]:

$$
\left[\begin{array}{l}
V_{n} \\
I_{n}
\end{array}\right]=\left[\begin{array}{ll}
A & B \\
C & D
\end{array}\right]\left[\begin{array}{l}
V_{n+1} \\
I_{n+1}
\end{array}\right] .
$$

For a symmetric structure $(A=D)$, the complex propagation constant $\gamma$ can be calculated as

$$
\cosh (\gamma p)=A \text {. }
$$


TABLE I

Circuit Elements for DifFerent Dielectric CoefFicients. The DIMENSIONS ARE $w=d=5 \mathrm{~mm}, h=0.8 d a=d 0.4$, AND $t=0.02 \mathrm{~mm}$ [FIG. 1(b)]

\begin{tabular}{|l|c|c|c|c|}
\hline & $\begin{array}{c}\text { Case 1: } \\
\varepsilon_{\mathrm{r}}=1\end{array}$ & $\begin{array}{c}\text { Case 2: } \\
\varepsilon_{\mathrm{r}}=2\end{array}$ & $\begin{array}{c}\text { Case 3: } \\
\varepsilon_{\mathrm{r}}=3\end{array}$ & $\begin{array}{c}\text { Case 4: } \\
\varepsilon_{\mathrm{r}}=4\end{array}$ \\
\hline$L_{1}$ & $0.75 \mathrm{nH}$ & $0.75 \mathrm{nH}$ & $0.75 \mathrm{nH}$ & $0.75 \mathrm{nH}$ \\
\hline$L_{2}$ & $0.2 \mathrm{nH}$ & $0.2 \mathrm{nH}$ & $0.2 \mathrm{nH}$ & $0.2 \mathrm{nH}$ \\
\hline$L_{3}$ & $0.82 \mathrm{nH}$ & $0.82 \mathrm{nH}$ & $0.82 \mathrm{nH}$ & $0.82 \mathrm{nH}$ \\
\hline$C_{1}$ & $15 \mathrm{fF}$ & $30 \mathrm{fF}$ & $45 \mathrm{fF}$ & $60 \mathrm{fF}$ \\
\hline$C_{2}$ & $94 \mathrm{fF}$ & $188 \mathrm{fF}$ & $282 \mathrm{fF}$ & $376 \mathrm{fF}$ \\
\hline$C_{3}$ & $63 \mathrm{fF}$ & $126 \mathrm{fF}$ & $189 \mathrm{fF}$ & $252 \mathrm{fF}$ \\
\hline
\end{tabular}

Another important parameter that can be extracted from the circuit elements is Bloch impedance. The Bloch impedance is the characteristic impedance of the TL periodically constructed by the SSP unit cells. This parameter ensures the compatibility of the SW-TL with other transmission lines:

$$
Z_{\text {in }}=Z_{0} \frac{V_{n+1}}{I_{n+1}}=\frac{Z_{0} B}{\sqrt{A^{2}-1}} .
$$

The transmission parameters can be calculated from the measured scattering parameters [27].

Fig. 1(a) depicts one unit cell of an SSP structure given in [4] along with the proposed equivalent circuit. The dimensions of the unit cell are specified in Fig. 1(b). The equivalent inductors and capacitors are calculated in static electromagnetics. Each current path of one unit cell can be modeled as an inductor [26] with the inductance of

$$
L_{i}=\frac{\mu_{0} l_{i}}{2 \pi}\left(\ln \frac{2 l_{i}}{w_{i}+t}+\frac{1}{2}\right)
$$

where $w, t$, and $l$ are width, thickness, and length of the metallic path for the current, respectively. It is obvious that decreasing the width and increasing the length of the current path increase the equivalent inductance. As the thickness is much smaller than the width, changing the thickness hardly affects any equivalent inductor. $L_{1}, L_{2}$ and $L_{3}$ as shown in Fig. 1(a) are calculated using (5). Fig. 1(c) specifies the geometries of the inductors of the unit cell. In this figure, the length of the $L_{3}\left(l_{3}\right)$ is equal to half width of the SSP unit cell, i.e., $w$ as shown in Fig. 1(b).

There are two types of equivalent capacitors in the proposed model. One consists of each pair of metallic plates $\left(C_{1}\right)$. This capacitor can be calculated by electrostatic software such as CST EM Studio. It is worthy to mention that $C_{1}$ includes part of the neighboring cells as depicted in Fig. 1(d). Simply, the capacitance increases as increasing the area of the plate and decreasing the distance between two plates. The distance between two plates $\left(d_{\mathrm{e}}\right)$ is equal to the period length of the structure $(d)$.

The other equivalent capacitor corresponds to the connection of the proposed structure to the ground $\left(C_{2}\right.$ and $\left.C_{3}\right)$. Each single metallic plate in electrostatics has a capacitance due to the electric fields from the structure that end at infinity. As no ground plane exists around the SSP structure, the accurate calculation of these elements is of great importance. For each capacitor, the capacitance can be calculated by EM software and measuring the capacitance of a single separate as well as a pair of metallic plates with the same geometries, as indicated in Fig. 1(d). Table I presents the calculated circuit element values for the SSP with four background media of different dielectric constants. All the inductances are identical because any change in permittivity does not affect the inductors. However, the capacitance linearly changes with the dielectric constant. Therefore, the capacitance for each medium is equal to the capacitance of the reference medium with $\varepsilon_{\mathrm{r}}=1$ multiplied by the relative permittivity of that medium. To understand the capacitance of single plates, one may consider simpler cases of circular metallic discs with the same areas as our rectangular $C_{2}$ and $C_{3}$. For a metallic circular disc of the radius $R$ and the area of $A$, the capacitance is [28]

$$
C=8 \varepsilon R=8 \varepsilon \sqrt{A / \pi} .
$$

Thus, the capacitance is proportional to the square root of the area of the metallic plate. For Case $1, C_{2}$ and $C_{3}$ from (6) are calculated to be 98 and $63 \mathrm{fF}$, which are very close to the simulated values by CST in Table I.

The dispersion curves from the equivalent circuit model which was solved by ADS, indicated as E. C., and the eigenmode solver of CST Microwave Studio indicated as CST are plotted in Fig. 1(e). The results show that the cutoff frequency is higher for the media with lower permittivity. Excellent agreement between the two methods demonstrates the accuracy of the proposed equivalent circuit model. The difference between the results from two methods may be caused by two main reasons. First, the equivalent elements are calculated in electrostatics and general definitions of voltage and current have been applied, which are not accurate at high frequencies, secondly, the currents and charge densities considered for the elements are slightly different from the real values at the periodic comb structure. For example, for a capacitor, we consider a constant voltage for the metal plate that is not very true at high frequencies. In order to improve the model, one can divide the unit cells into even smaller pieces in order to account for the nonuniform voltage and current distribution. However, the model will be much more complicated and consequently not desirable for further engineering design purposes. The current simple model provides a simple and viable tool for designing SSP structures for specific frequency ranges.

Another adjustable parameter in the equivalent circuit model is the Bloch impedance in (4). By tuning the parameters to achieve $50-\Omega$ impedance, the structure will be more compatible with a conventional transmission line while it is surely quite straightforward to tune the structure to achieve other impedances. The SSP structure is studied from the Bloch impedance point of view. The simulation has been done for the SSP printed on a piece of Rogers $4003(1.52 \mathrm{~mm})$ slab with an effective dielectric constant of $\varepsilon_{\mathrm{r}}=2$ used in our experiment. The effective permittivity for this Rogers substrate is calculated by CST Microwave Studio. For this purpose, the dispersion curves of the structure for two cases are compared: the SSP structure on Rogers 4003 (as a partially filled medium) and the SSP structure in the fully filled medium with the effective permittivity. Our calculation showed an almost constant Bloch impedance of $42 \Omega$ over a wide frequency range for the SSP structure for the 
medium with $\varepsilon_{\mathrm{r}}=2$ as a function of frequency as shown in the inset of Fig. 1(e). These results confirm that this structure can be matched to any $50-\Omega$ transmission line.

With high field confinement and the constant input impedance of $\sim 50 \Omega$ for a wide range of frequencies, the SSP structures are able to provide a plausible option for inexpensive and small transmission lines. The next step is to design an appropriate transition to connect this potential transmission line to typical transmission lines and realize the SW-TL.

\section{MODE CONVERSION}

In order to exploit the high field confinement and groundless nature of the SSP structure in microwave circuits, the structure should be connected to conventional transmission lines, which can result in wider applications and better compatibility of the proposed SSP structures. In order to obtain highly efficient connection, it is necessary to match two transmission structures in terms of impedance, polarization and momentum. Based on the proposed design, the impedance of the SSP structure was designed around $50 \Omega$, and, therefore, no more impedance matching is needed.

Fig. 2(a) presents the SSP structure connected to a microstrip line through the proposed mode converter. The microstrip line supports the quasi-TEM modes, and these modes should be transformed to TM polarized SSPs. Fig. 2(b) and (c) shows the normal $\left(\left|E_{\mathrm{z}}\right|\right)$ and azimuthal $\left(\sqrt{ }\left(\left|E_{\mathrm{x}}\right|^{2}+\left|E_{\mathrm{y}}\right|^{2}\right)\right)$ magnitudes of electrical field at planes perpendicular to the mode converter, respectively. These results clearly demonstrate the gradual transformation from quasi-TEM modes to TM modes via the proposed mode converter (at $7 \mathrm{GHz}$ ). It can be observed from Fig. 2 that the normal component of quasi-TEM waves at a microstrip line (as shown in Fig. 2(b), Plane 1) is almost zero, and the major part of the electric field of the microstrip line is azimuthal component (Fig. 2(c), Plane 1). Fig. 2(b) depicts a gradual increase in the normal component of the electrical field via the mode converter. This component becomes the major electrical field at the SSP structure as shown in both Fig. 2(b) and (c), Plane 6.

The proposed mode converter acts as a momentum converter while it transforms the polarization. The microstrip lines support the waves with a wavenumber equal to that of the light $\left(k_{0}\right)$; however, the dispersion curve for the SSP structure [Fig. 1(e)]indicates that its wavenumber is much larger than $k_{0}$. Momentum conversion is achieved by gradually increasing the wavenumber as the wave propagates through the mode converter. Fig. 2(d) presents the dispersion curve at various points along the mode converter. These curves are obtained by considering a periodic configuration of each section of the mode converter separately. These separate sections are specified in the inset of Fig. 2(d). This figure clarifies the gradual increase of the momentum of the wave propagating through the mode converter.

\section{Slow-Wave Transmission Line}

Next, the SSP structure is connected to a conventional TL. In this paper, we first present the microstrip line mode converter to the SSP. The mode converter is designed in order to connect the SSP unit cells to a $50-\Omega$ microstrip line on a piece of $1.52-\mathrm{mm}$
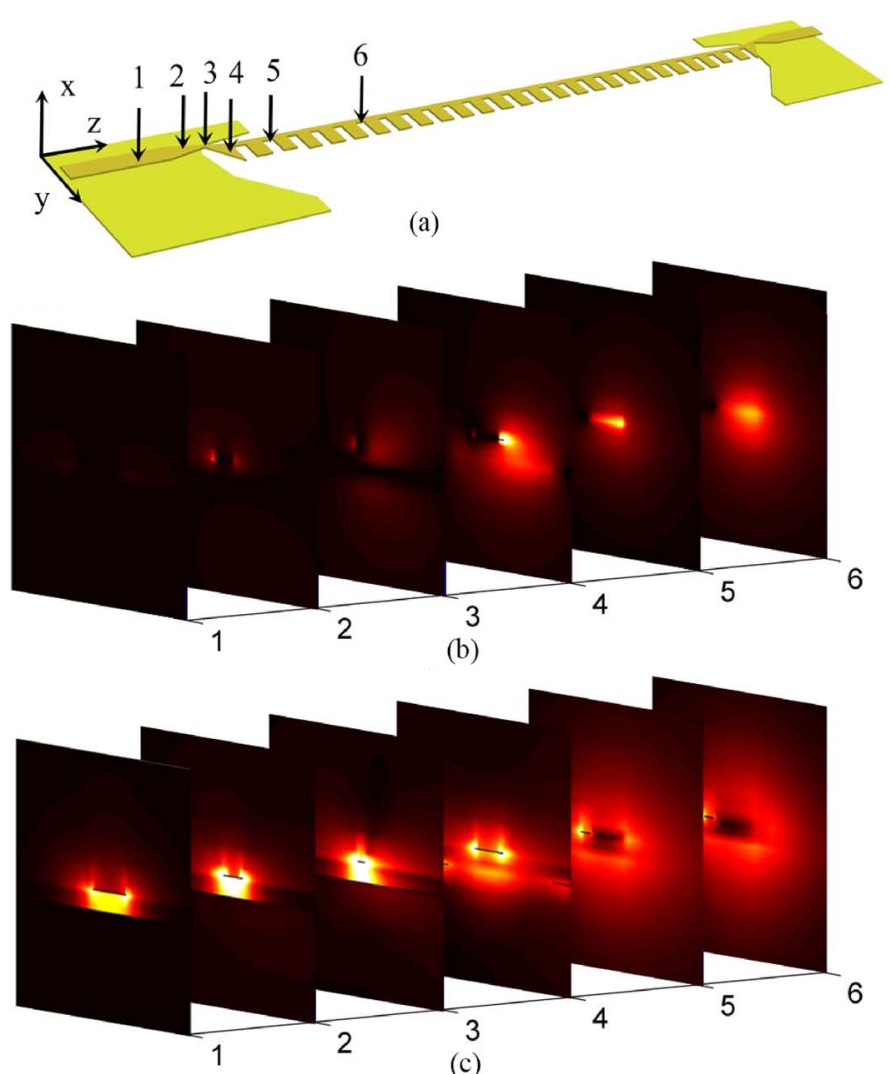

(c)

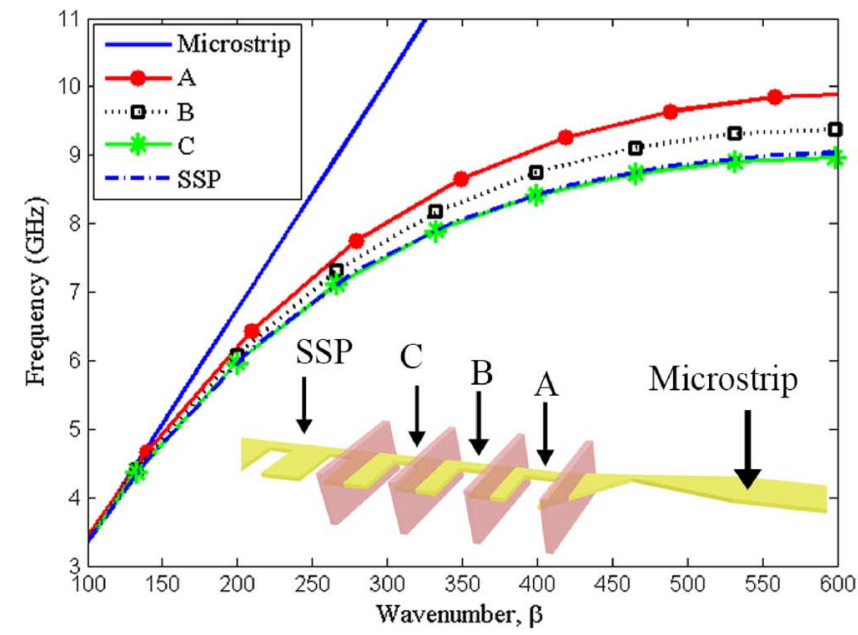

(d)

Fig. 2. (a) Proposed SSP structure connected to the mode converter to construct the SW-TL. (b) Normal and (c) azimuthal components of the electrical field at six perpendicular planes to the mode converter. (d) Dispersion curves of the separate parts of the mode converter. Inset: The separate parts of the mode converter.

Rogers 4003 substrate to construct the SW-TL for the reflection lower than $-12 \mathrm{~dB}$

\section{A. Constructing the Structure}

The mode converter is composed of two parts: the strip connector and the ground regulator as shown in Fig. 3(a). These two parts are designed based on the parameters of the microstrip line and the SSP unit cell. The dimensions of the SSP are $w$ $=d=5 \mathrm{~mm}, h=0.8 d$, and $a=0.4 d[$ Fig. 1(b)]. To achieve 


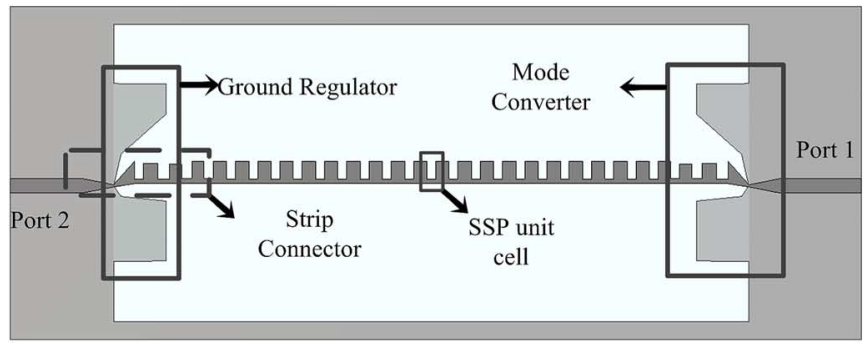

(a)

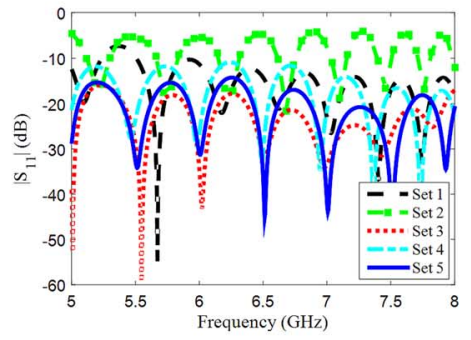

(e)

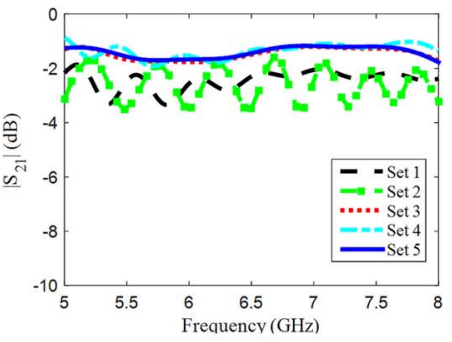

(d)

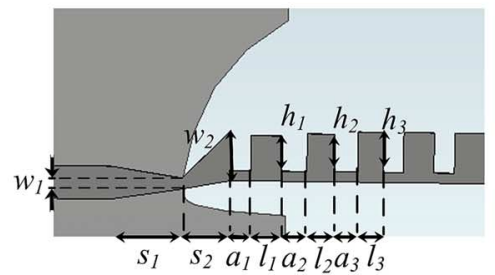

(b)

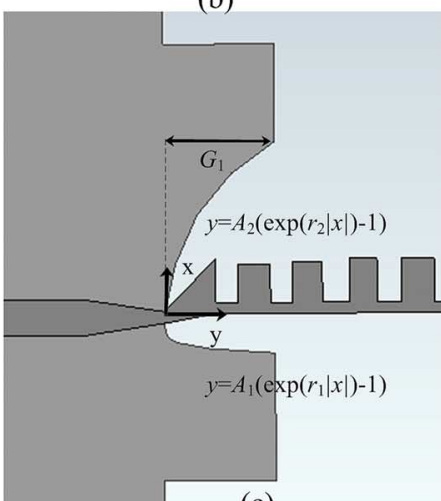

(c)

Fig. 3. (a) SSP structure connected to the microstrip line via matching transitions to build the SW-TL. (b) Specified parameters for the strip connector. (c) Specified parameters for the ground regulator. (d) Simulated $\left|S_{21}\right|$ and (e) $\left|S_{11}\right|$ for five parameter sets (Table III) for the entire frequency range.

$50-\Omega$ impedance for the design on Rogers $40031.52 \mathrm{~mm}$, the microstrip line width is set as $w_{s}=3.2 \mathrm{~mm}$. Fig. 1(d) shows that the cutoff frequency $\left(f_{\mathrm{c}}\right)$ for the SSP structure on 1.52-mm Rogers 4003 substrate with the effective epsilon of 2 is $9.1 \mathrm{GHz}$. This frequency can be changed by scaling the SSP dimensions. Here, we design the mode converter to efficiently operate at the bandwidth of $5-8 \mathrm{GHz}$. All of the parameters are presented with respect to the period length $(d)$. Consequently, the working frequency range for both the mode converter and the SSP structure can be scaled by changing the period length of the SSP unit cells.

1) Constructing the Strip Connector: Fig. 3(b) shows the strip connector. This design includes three SSP unit cells. The effect of the number of unit cells on the performance of the converter will be discussed later. The parameters of the strip connectors are specified in Fig. 3(b). The parameters are related to each other based on the formulas given in Table II. These relations can be applied to design the transitions for SSPs working at other frequency ranges. In the following, we explain the relation between the parameters in detail. The width of the strip decreases from $w_{\mathrm{s}}$ to $w_{1}=w_{\mathrm{s}} / x$ and then increases to $w_{2}=w$. These changes have two effects to the fields. First, it helps the transformation of the polarization (Fig. 2(b) from Planes 1 to 4). Second, the increase in the strip width from $w_{1}$ to $w_{2}$ compensates for the increase in the distance between the ground and the strip where the ground is gradually removed. This keeps the impedance of the line almost the same for the rest of the microstrip guiding modes before their complete transformation to the SSP modes and therefore reduces their reflection. $S_{1}$ and $S_{2}$ are the required lengths for the gradual width change and are chosen based on the equations in Table II. $h_{1}, h_{2}, h_{3}, a_{1}, a_{2}$, $a_{3}, l_{1}, l_{2}$, and $l_{3}$ are the dimensions of the three separate parts of the strip connector, as shown in the inset of Fig. 2(d), and are chosen to achieve a gradual momentum increase, as depicted in Fig. 2(d), and the optimized relations for the best performance
TABLE II

Relation Between Parameters of the Strip Connector [Fig. 3(b)]

\begin{tabular}{|c|c|c|}
\hline$h_{1}=h-2 \times \Delta h$ & $a_{1}=a$ & $l_{1}=l=d-a$ \\
$h_{2}=h-1 \times \Delta h$ & $a_{2}=a+2 \times \Delta a$ & $l_{2}=l-1 \times \Delta l$ \\
$h_{3}=h$ & $a_{3}=a+1 \times \Delta a$ & $l_{3}=l-2 \times \Delta l$ \\
& & $\Delta l=\Delta a$ \\
\hline$S_{1}=1.5 \times d-\Delta S$ & $w_{1}=w_{\mathrm{s}} / x$ & \\
$S_{2}=d-\Delta S$ & $w_{2}=w$ & \\
\hline
\end{tabular}

of these parameters are given in Table II. Referring to Table II, the parameters which are critical to design the strip connector include: $\Delta h, \Delta a, \Delta S$, and $x$.

2) Constructing the Ground Regulator: This part regulates the discontinuity of the ground plane and is located where the ground is removed. On both sides of the strip, the edges of the ground regulator have the functionality of $y=A\left(\mathrm{e}^{r|x|}-1\right)$ as shown in Fig. 3(c), where $r$ and $A$ are two selected parameters. One can select the initial values so that the edges of the ground regulator follow the change of the strip connector properly. For simplicity, we chose $A_{1}$ and $A_{2}$ to be $d / 500(0.01 \mathrm{~mm})$ and $d / 5(1 \mathrm{~mm})$ for the two sides, respectively and further selectivity can be achieved by tuning $r$ for the best $S$-parameter results. The ground regulator truncates at the half length of the strip connector as follows:

$$
G_{1}=0.5\left(S_{2}+a_{1}+l_{1}+a_{2}+l_{2}+a_{3}+l_{3}\right) .
$$

Consequently, the parameters of the ground regulator are found to be $r_{1}$ and $r_{2}$.

\section{B. Tuning and Optimization}

In order to achieve high transmission and low reflection, we tune $\Delta h, \Delta a, \Delta S, x, r_{1}$, and $r_{2} . \Delta h, \Delta a$, and $\Delta S$ should be small fractions of $d$. Here, $\Delta h=\Delta a=\Delta S=d / 20$ (or $0.25 \mathrm{~mm}$ ) are chosen. $x$ determines the strip width reduction, 


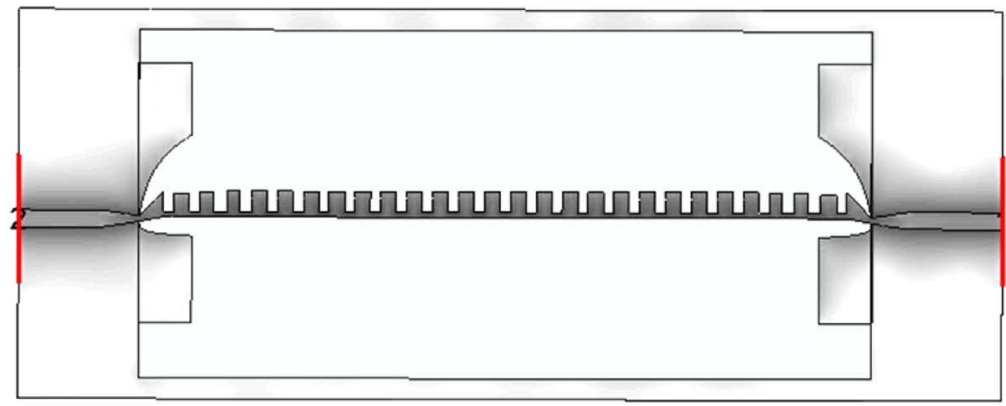

(a)

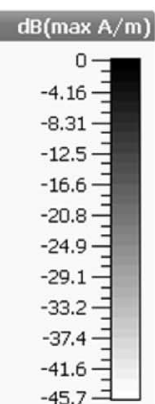

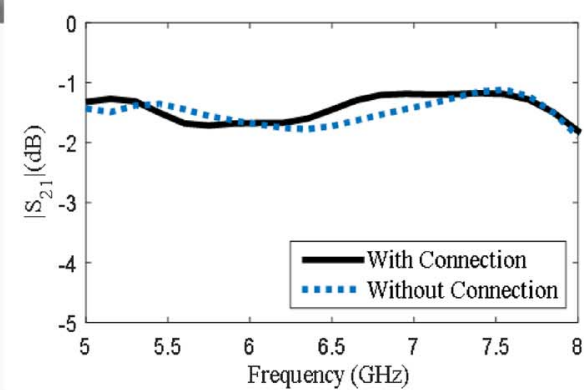

(b)

Fig. 4. (a) Surface current on the SW-TL at 7 GHz. (b) Comparison of the $S$-parameter results (simulation) of SW-TL with and without port connection.

TABLE III

Five Parameter Sets in Millimeters for the Mode Converter

\begin{tabular}{|l|l|l|l|l|l|}
\hline & Set 1 & Set 2 & Set 3 & Set 4 & Set 5 \\
\hline$\Delta h$ & $d / 20$ & $d / 20$ & $d / 20$ & 0 & $d / 20$ \\
\hline$\Delta a$ & $d / 20$ & $d / 20$ & 0 & $d / 20$ & $d / 20$ \\
\hline$\Delta S$ & $d / 20$ & $d / 20$ & 0 & $d / 20$ & $d / 20$ \\
\hline$x$ & 3 & 1 & 3 & 3 & 3 \\
\hline$r_{1}$ & $5 / d$ & $10.5 / d$ & $10.5 / d$ & $10.5 / d$ & $10.5 / d$ \\
\hline$r_{2}$ & $0.5 / d$ & $0.75 / d$ & $0.75 / d$ & $0.75 / d$ & $0.75 / d$ \\
\hline
\end{tabular}

and its initial value can be kept as 3 for designing the transition to any SSP structure. $r_{1}$ and $r_{2}$ determine the shape of the ground regulator and the initial values are chosen as $10.5 / d$ (or $2.1 \mathrm{~mm}^{-1}$ ) and $0.75 / d$ (or $0.15 \mathrm{~mm}^{-1}$ ). Fig. 3(d) and (e) study the effect of these geometric parameters by comparing the $S$-parameter results for five parameter sets listed in Table III. As can be seen, with Set 5 , the SW-TL achieves the best transmission performance among the five sets.

Fig. 4(a) presents the absolute values of the surface current on the metallic surfaces of the structure at $7 \mathrm{GHz}$. It can be seen that gradually removing the ground at the connection of the SSP structure and microstrip line (the matching part on the ground plane) prevents the discontinuity of the surface currents and consequently reduces the reflection maybe caused by this abrupt change.

Both ports of the designed SW-TL have common ground as they are connected by a metallic connection as shown in Fig. 4(a). Fig. 4(a) shows that the current density on this metallic bar is at least $25 \mathrm{~dB}$ smaller than the maximum current density at the SW-TL. In order to ensure that this metallic connection does not have any significant effect on the performance of the groundless transmission line, the proposed SW-TL without any ground connection is simulated and the results are compared in Fig. 4(b) with the SW-TL with port connection. The results indicate that this connection does not change the transmission significantly.

The strip connector consists of straight lines. We anticipate better results by substituting the curve lines of ground regulator by straight lines as it is shown in Fig. 5(a). $G_{2}=d / 2.8=$ $1.8 \mathrm{~mm}$ is tuned for good transmission. For better performance, "Interpolated Quasi Newton" optimization method is performed on the whole parameters. Fig. 5(b) compares the results from the both SW-TLs with curved and straight ground regulators as well as the results from final optimization. The parameters after final optimization are presented in Table IV.
TABLE IV

PARAmeter VAlues of SW-TL (Fig. 3(b) AND (c)) After Optimization (IN MILLIMETERS)

\begin{tabular}{|l|l|l|l|l|l|}
\hline$h_{1}$ & 3.5 & $a_{1}$ & 2 & $l_{1}$ & 3.2 \\
\hline$h_{2}$ & 3.4 & $a_{2}$ & 2.5 & $l_{2}$ & 2.8 \\
\hline$h_{3}$ & 3.8 & $a_{3}$ & 2.3 & $l_{3}$ & 2.7 \\
\hline$w_{1}$ & 0.5 & $S_{1}$ & 7 & $G_{1}$ & 10.1 \\
\hline$w_{2}$ & 5 & $S_{2}$ & 4.7 & & \\
\hline
\end{tabular}

The ideal transition should be composed of an infinite number of gradually changing SSP elements; however, we should find the most effective transition with as few elements as possible. The strip connector in Fig. 3(a) is composed of three SSP elements. The most important role of these elements is to gradually increase the momentum and as it is indicated in Fig. 2(d), three elements can provide smooth momentum matching and consequently, increasing the number of matching elements does not lead to better transmission and reflection. Fig. 5(c) compares the $S$-parameter results for four types of mode converters: single-element, two-element, three-element, and four-element strip connectors. The results show that the strip connector with three elements has better performance than single-element and two-element types. However, the transmission is almost the same as that of four-element type. Consequently, due to its shorter length, the three-element mode converter is chosen.

\section{Results AND Discussion}

The $S$-parameters were measured by a vector network analyzer. Fig. 5(d) depicts the fabricated SW-TL connected to the vector network analyzer. The results are presented in Fig. 5(e). $\left|S_{21}\right|$ for the SW-TL is calibrated based on the $50-\Omega$ microstrip line, in order to cancel out the connection loss and dielectric loss as well as to study the role of the mode converter and the SSP structure on the transmission. The difference between the measured and simulated $\left|S_{11}\right|$ is attributed to the effect of SMA connectors, which is not considered in the simulation. The results confirm that the experimental and simulated results agree well with each other.

The experimental and simulation results show about $-1.5 \mathrm{~dB}$ insertion loss for the SW-TL. This insertion loss includes the conversion loss ( $L_{\text {con }}$ by the mode converter) and the transmission loss ( $L_{t}$ by the SSP cells) as

$$
\text { Loss }=L_{\text {con }}+L_{t} \text {. }
$$




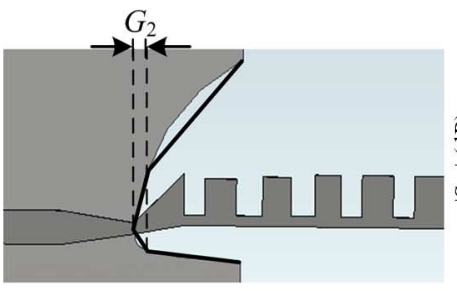

(a)

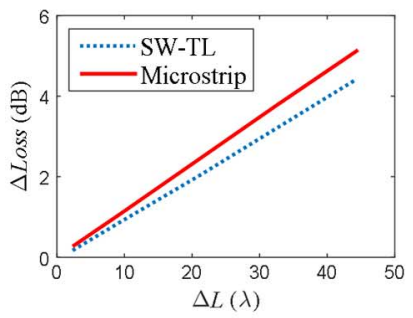

(g)

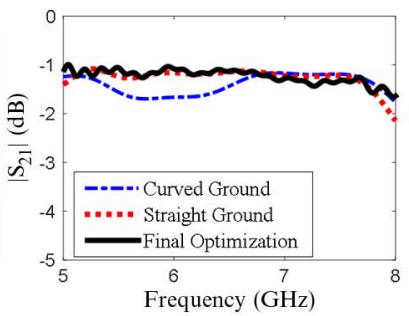

(b)

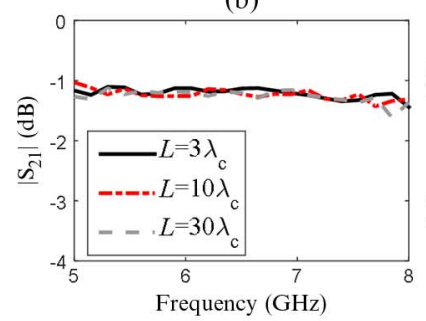

(f)

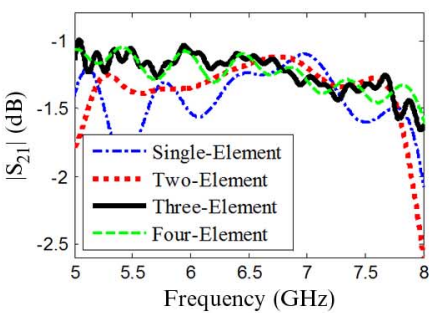

(c)

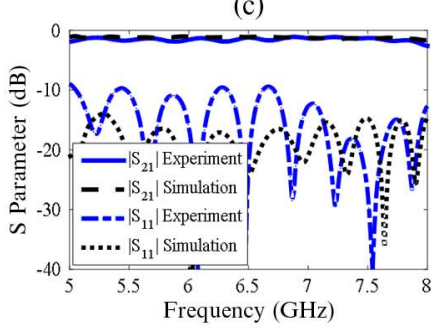

(e)

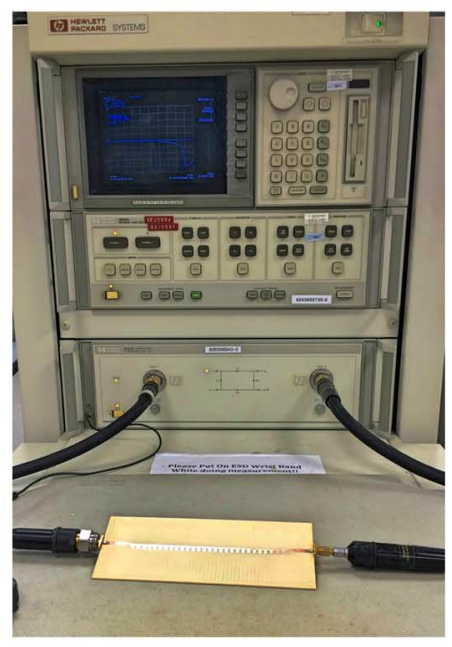

(d)

Fig. 5. (a) Substitution of curved lines of the ground regulator by straight lines. (b) $S$-parameter results (simulation) of SW-TL with curved ground regulator, straight ground regulator and SW-TL with parameters given in Table IV (c) $S$-parameter results (simulation) for SW-TL with single-, two-, three-, and four-unitcells strip connectors (d) the proposed SW-TL connected to the vector network analyser. (e) Comparison between experiment and simulation of the $S$-parameters for the proposed structure. (f) $S$-parameter results (simulation) for SW-TLs with various lengths on a lossless dielectric. (g) Insertion loss increases with increasing the total length for the designed SW-TL and an ideal microstrip line on a lossy dielectric (simulation results).

The transmission loss is composed of radiation loss $\left(L_{\mathrm{Rad}}\right)$ and dielectric loss $\left(L_{d}\right)$ as follows:

$$
L_{\mathrm{t}}=L_{\mathrm{Rad}}+L_{d}
$$

To study the radiation loss, we simulate the SW-TLs on a lossless dielectric with different lengths. Fig. 5(f) depicts the $S$-parameter results ( $L$ is the total length and in terms of the cutoff wavelength). The simulation results show that, for a lossless dielectric, the insertion loss does not increase by increasing the length of the transmission line. This fixed loss is mainly caused by the mode converter $\left(L_{\text {con }}\right)$. In other words, the radiation loss $\left(L_{\mathrm{Rad}}\right)$ from the single-line SW-TL is negligible. Fig. 5(g) compares the transmission loss for the SW-TL on a lossy Rogers $40031.52 \mathrm{~mm}$ with a microstrip line on the same dielectric layer. To exclude the conversion loss, this figure presents the insertion loss increase ( $\Delta$ Loss) by increasing the length of the transmission line $(\Delta L) . \Delta L$ is presented in terms of the free space wavelength $(\lambda)$ at the operating frequency $(7$ $\mathrm{GHz}$ ). This figure shows that for SW-TL, the dielectric loss $\left(L_{d}\right)$ increases by as much as $9.6 \mathrm{~dB}$ per $100 \lambda$, which is smaller than $11.6 \mathrm{~dB} / 100 \lambda$ loss for microstrip line. The reason for the smaller transmission loss is that, compared with the microstrip line, smaller portion of the fields are distributed in the dielectric below SW-TL and consequently, smaller portion of the field interacts with the lossy dielectric. Small transmission loss along with its low profile and planar structure empowers the great potential of SW-TL for serving as a low-loss and effective groundless transmission line in microwave systems.

\section{CONCLUSION}

An equivalent circuit model for spoof surface plasmon structures has been first proposed as a viable tool for analyzing and designing the structure with desired Bloch impedance and cutoff frequency. The equivalent circuit has demonstrated the constant and approximately $50-\Omega$ input impedance of the spoof surface plasmon structure and therefore confirmed the possibility of a transmission line with SSP structures. A mode converter has been presented to convert the polarization and momentum of spoof surface plasmons in order to couple with the quasi-TEM modes of microstrip lines. The scattering parameters of the proposed SW-TL have been measured and the high transmission and low reflection have confirmed the excellent performance of the proposed design, which can bring groundbreaking technologies of groundless multilayer microwave circuits to the RF engineering and daily-life applications.

\section{ACKNOWLEDGMENT}

The authors would like to thank H. Ye, National University of Singapore, for his help in preparing the figures. The authors would also like to thank CST Inc. for donating the CST EM-Studio software license.

\section{REFERENCES}

[1] W. L. Barnes, A. Dereux, and T. W. Ebbesen, "Surface plasmon subwavelength optics," Nature, vol. 424, no. 6950, pp. 824-830, 2003.

[2] J. M. Pitarke, V. M. Silkin, E. V. Chulkov, and P. M. Echenique, "Theory of surface plasmons and surface-plasmon polaritons," Rep. Prog. Phys., vol. 70, no. 1, pp. 1-88, 2007.

[3] J. B. Pendry, L. Martin-Moreno, and F. J. Garcia-Vidal, "Mimicking surface plasmons with structured surfaces," Science, vol. 305, no. 5685, pp. 847-848, 2004.

[4] X. Shen, T. J. Cui, D. Martin-Cano, and F. J. Garcia-Vidal, "Conformal surface plasmons propagating on ultrathin and flexible films," Proc. Nat. Acad. Sci., vol. 110, no. 1, pp. 40-45, 2013.

[5] M. Navarro-Cía, M. Beruete, S. Agrafiotis, F. Falcone, M. Sorolla, and S. A. Maier, "Broadband spoof plasmons and subwavelength electromagnetic energy confinement on ultrathin metafilms," Opt. Exp., vol. 17, no. 20, pp. 18184-18195, 2009.

[6] D. Martin-Cano, O. Quevedo-Teruel, E. Moreno, L. Martin-Moreno, and F. J. Garcia-Vidal, "Waveguided spoof surface plasmons with deep-subwavelength lateral confinement," Opt. Lett., vol. 36, no. 23, pp. 4635-4637, 2011. 
[7] M. Navarro-Cía, M. Beruete, M. Sorolla, and S. A. Maier, "Enhancing the dual-band guiding capabilities of coaxial spoof plasmons via use of transmission line concepts," Plasmonics, vol. 6, no. 2, pp. 295-299, 2011.

[8] X. Gao, J. H. Shi, X. Shen, H. F. Ma, W. X. Jiang, L. Li, and T. J. Cui, "Ultrathin dual-band surface plasmonic polariton waveguide and frequency splitter in microwave frequencies," Appl. Phys. Lett., vol. 102, 2013, Art. ID 151912.

[9] X. Gao, L. Zhou, Z. Liao, H. F. Ma, and T. J. Cui, "An ultra-wideband surface plasmonic filter in microwave frequency," Appl. Phys. Lett., vol. 104, no. 19, 2014, Art. ID 191603.

[10] X. Liu, Y. Feng, B. Zhu, J. Zhao, and T. Jiang, "High-order modes of spoof surface plasmonic wave transmission on thin metal film structure," Opt. Exp., vol. 21, no. 25, pp. 31155-31165, 2013.

[11] S. Pandey, B. Gupta, and A. Nahata, "Terahertz plasmonic waveguides created via 3D printing," Opt. Exp., vol. 21, no. 21, pp. 24422-24430, 2013.

[12] G. Goubau, "Open wire lines," IRE Trans. Microw. Theory Techn., vol. MTT-4, no. 4, pp. 197-200, Apr. 1956.

[13] A. Treizebré, T. Akalin, and B. Bocquet, "Planar excitation of Goubau transmission lines for THz bioMEMS," IEEE Microw. Wireless Compon. Lett., vol. 15, no. 12, pp. 886-888, Dec. 2005.

[14] T. Akalin, A. Treizebré, and B. Bocquet, "Single-wire transmission lines at terahertz frequencies," IEEE Trans. Microw. Theory Techn., vol. 54, no. 6, pp. 2762-2767, Jun. 2006

[15] T. Akalin, E. Peytavit, and J. F. Lampin, "THz long range plasmonic waveguide in membrane topology," in Proc. 33rd Int. Conf. in Infrared, Millimeter and Terahertz Waves, 2008, pp. 1-2.

[16] T. Akalin and W. Padilla, "Plasmonic waveguides and metamaterial components at terahertz frequencies," in Proc. Asia-Pacific Microw. Conf., 2009, pp. 2444-2446.

[17] Y. Xu, C. Nerguizian, and R. G. Bosisio, "Wideband planar Goubau line integrated circuit components at millimetre waves," IET Microw. Antennas Propagat., vol. 5, no. 8, pp. 882-885, 2011

[18] J. Emond, M. Grzeskowiak, G. Lissorgues, S. Protat, F. Deshours, E. Richalot, and O. Picon, "A low-loss planar goubau line and a coplanar-PGL transition on high-resistivity silicon substrate in the 57-64 GHz band," Microw. Opt. Technol. Lett., vol. 54, no. 1, pp. 164-168, 2012.

[19] S. Laurette, A. Treizebre, and B. Bocquet, "Corrugated Goubau lines to slow down and confine THz waves," IEEE Trans. THz Sci. Technol., vol. 2, no. 3, pp. 340-344, May 2012.

[20] D. Sanchez-Escuderos, M. Ferrando-Bataller, J. I. Herranz, and M. Cabedo-Fabres, "Periodic leaky-wave antenna on planar Goubau line at millimeter-wave frequencies," IEEE Antennas Wireless Propag. Lett., vol. 12, pp. 1006-1009, 2013.

[21] H. F. Ma, X. Shen, Q. Cheng, W. X. Jiang, and T. J. Cui, "Broadband and high-efficiency conversion from guided waves to spoof surface plasmon polaritons," Laser Photon. Rev., vol. 8, no. 1, pp. 146-151, 2013.

[22] L. Liu, Z. Li, C. Gu, P. Ning, B. Xu, Z. Niu, and Y. Zhao, "Multichannel composite spoof surface plasmon polaritons propagating along periodically corrugated metallic thin films," J. Appl. Phys., vol. 116, no. 1, 2014, Art. ID 013501.

[23] B. C. Pan, Z. Liao, J. Zhao, and T. J. Cui, "Controlling rejections of spoof surface plasmon polaritons using metamaterial particles," Opt. Exp., vol. 22, no. 11, pp. 13940-13950, 2014.

[24] X. Liu, Y. Feng, K. Chen, B. Zhu, J. Zhao, and T. Jiang, "Planar surface plasmonic waveguide devices based on symmetric corrugated thin film structures," Opt. Exp., vol. 22, no. 17, pp. 20107-20116, 2014.

[25] J. Papapolymerou, G. E. Ponchak, E. Dalton, A. Bacon, and M. M. Tentzeris, "Crosstalk between finite ground coplanar waveguides over polyimide layers for 3-D MMICs on $\mathrm{Si}$ substrates," IEEE Trans. Microw. Theory Techn., vol. 52, no. 4, pp. 1292-1301, Apr. 2004

[26] Z. Piatek, B. Baron, T. Szczegielniak, D. Kusiak, and A. Pasierbek, "Self inductance of long conductor of rectangular cross section," Przeglad Elektrotechniczny Electr. Rev., vol. 88, no. 8, pp. 323-326, 2012.

[27] D. M. Pozar, Microwave Engineering, 3rd ed. New York, NY, USA Wiley, 2004.

[28] J. D. Jackson, Classical Electrodynamics. New York, NY, USA: Wiley, 1975, vol. 3, p. 128, problem 3.3.

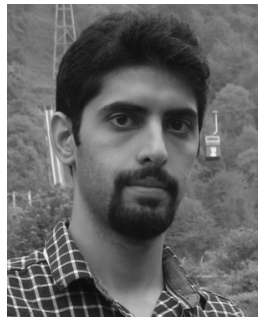

Amin Kianinejad (S'14) received the B.Sc. degree from the Shiraz University of Technology, Shiraz, Iran, and the M.Sc. degree from Sharif University, Tehran, Iran both in electrical engineering. He is currently working toward the Ph.D. degree at the National University of Singapore, Singapore.

His current research interests are microwave plasmonics, antennas, and metamaterials.

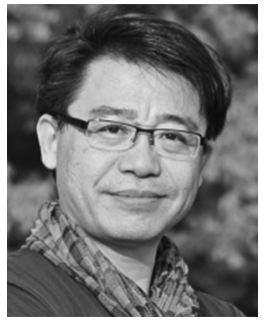

Zhi Ning Chen (M'99-SM'05-F'07) received the B.Eng., M.Eng., and Ph.D. degrees in electrical engineering from the Institute of Communications Engineering, Nanjing, China, and the Ph.D. degree from University of Tsukuba, Ibaraki, Japan.

During 1988-1995, he was a Lecturer and later an Associate Professor with the Institute of Communications Engineering, Nanjing, China, as well as at Southeast University as a Postdoctoral Fellow and later as an Associate Professor. During 1995-1997, he was with the City University of Hong Kong as a Research Assistant and later a Research Fellow. In 1997, he was awarded the Japan Society for the Promotion of Science (JSPS) Fellowship to conduct his research at the University of Tsukuba, Japan. In 2001 and 2004, he visited the University of Tsukuba under a JSPS Fellowship Program (senior level). In 2004, he was with IBM T. J. Watson Research Center, Yorktown Heights, NY, USA, as an Academic Visitor. During May-June 2013, he was a Senior DIGITEO Guest Scientist with the "Laboratoire des SignauxetSystèmes", UMR8506 CNRS-Supelec-University Paris Sud, Gif-sur-Yvette, France. During 1999-2012, he was with the Institute for Infocomm Research $\left(\mathrm{I}^{2} \mathrm{R}\right.$ ) (formerly known as Centre for Wireless Communications and Institute for Communications Research) as a Member of Technical Staff (MTS), Senior MTS, Principal MTS, Senior Scientist, Lead Scientist, and Principal Scientist as well as Head for RF \& Optical Department. In 2012, he joined the Department of Electrical and Computer Engineering, National University of Singapore, Singapore, as a Full Professor. $\mathrm{He}$ is concurrently holding a joint appointment as Advisor and Principle Scientist at $\mathrm{I}^{2} \mathrm{R}$ as well as professorships at Southeast University (Changjiang Guest Professor), Nanjing University (Guest Professor), Shanghai Jiaotong University (Guest Professor), Tsinghua University (Visiting Professor), Tongji University (Guest Professor), University of Science and Technology of China (Guest Professor), Fudan University (Outstanding Overseas Visiting Professor), Dalian Maritime University, Chiba University (Visiting Professor), National Taiwan University of Science and Technology (Visiting Professor), Shanghai University (Ziqiang Professor), Beijing University of Posts and Telecommunications (Visiting Professor), Tohoku University (Visiting Professor) as well as City University of Hong Kong (Adjunct Professor). He has published 480 technical papers and authored/edited the books Broadband Planar Antennas (Wiley 2005), UWB Wireless Communication (Wiley 2006), Antennas for Portable Devices (Wiley 2007), and Antennas for Base Stations in Wireless Communications (McGraw-Hill 2009). Currently, he is working on Handbook of Antenna Technologies (Springer References) as a Chief Editor. He has also contributed chapters to the books UWB Antennas and Propagation for Communications, Radar, and Imaging (Wiley 2006), Antenna Engineering Handbook (McGraw-Hill 2007), as well as Microstrip and Printed Antennas (Wiley 2010). He holds 30 patents and has 31 licensed deals with industry. His current research interest includes electromagnetic engineering, antennas for microwaves, $\mathrm{mmW}$, submmW, and $\mathrm{THz}$ communication, radar, imaging and sensing systems.

Dr. Chen was one of the founding General Chairs of International Workshop on Antenna Technology (iWAT), International Symposium on InfoComm \& Media Technology in Bio-Medical \& Healthcare Applications (IS 3T-in-3A), International Microwave Forum (IMWF) as well as Asia-Pacific Conference on Antennas and Propagation (APCAP). He was the recipient of International Symposium on Antennas and Propagation Best Paper Award 2010, the CST University Publication Award 2008, IEEE AP-S Honorable Mention Student Paper Contest 2008, Institution of Engineers Singapore (IES) Prestigious Engineering Achievement Awards 2006, 2013 and 2014, I ${ }^{2}$ R Quarterly Best Paper Award 2004, IEEE iWAT 2005 Best Poster Award, and several technology achievement awards from China during 1990-1997. He is currently serving as an associate editor for the IEEE TRANS ANTENNAS AND PROPAGATION and the IEEE Antennas and Propagation Society as a Distinguished Lecturer since 2009. 


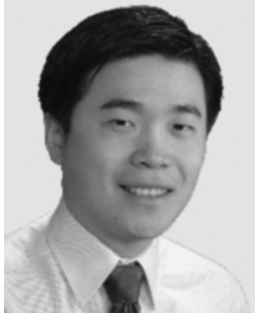

Cheng-Wei Qiu (S'03-M'08) received the B.Eng. degree from the University of Science and Technology of China, Hefei, China, in 2003, and the $\mathrm{Ph} . \mathrm{D}$. degree from the National University of Singapore, Singapore, in 2008.

He was a Postdoctoral Fellow with Physics Department, Massachusetts Institute of Technology, Cambridge, MA, USA, until the end of 2009. In December 2009, he joined the National University of Singapore (NUS), Singapore, as an Assistant Professor. He also held or is holding Visiting Professor positions with the University of Paris 11, KAUST, and Shenzhen University. He has published over 100 journal peer-reviewed papers and given three keynote presentations in international conferences. His research interests are in the areas of electromagnetic wave theory of transformation optic metamaterials, light-matter interaction, and nanophotonics.

Prof. Qui was the recipient of the SUMMA Graduate Fellowship in Advanced Electromagnetics in 2005, the IEEE Antennas and Propagation Society (AP-S) Graduate Research Award in 2006, the URSI Young Scientist Award in 2008, the NUS Young Investigator Award in 2011, the MIT TR35@Singapore Award in 2012, the Young Scientist Award by Singapore National Academy of Science 2013, and the Faculty Young Research Award in NUS 2013. He has served on editorial boards for various journals and as TPC chairman for various conferences. 